J. Clin. Chem. Clin. Biochem.

Vol. 25, 1987, pp. 493-498

(C) 1987 Walter de Gruyter \& Co.

Berlin - New York

\title{
Catalytic Activity Concentrations of Diadenosine Tetraphosphate Hydrolase in Normal and Pathological Sera
}

\author{
By J. Lüthje, A. Holler, A. Ogilvie \\ Institut für Physiologische Chemie der Universität Erlangen-Nürnberg \\ W. Siegfried
}

Medizinische Klinik der Universität Erlangen-Nürnberg

A. Waldherr

Zentrallaboratorium im Universitätskrankenhaus der Universität Erlangen-Nürnberg and

W. Domschke

Medizinische Klinik der Universität Erlangen-Nürnberg

(Received December 17, 1986/May 13, 1987)

Summary: The levels of diadenosine tetraphosphate hydrolase, a nucleotide pyrophosphatase (EC 3.6.1.9), were measured in human sera with a bioluminescence method. 40 sera of healthy donors and 207 samples obtained from inpatients of a medical clinic were analysed. About two thirds of the patients showed increased hydrolase levels, as compared to the normal donors. Elevated levels were not specific for a certain disease, and they were found in quite different disorders. There was no indication for a dependency of enzyme activity on age or sex of the patients. The comparison of diadenosine tetraphosphate hydrolase with seven other laboratory parameters revealed a highly significant correlation with $\gamma$-glutamyl transferase, suggesting that the liver may be a source of elevated serum activities. There was evidence that the gastrointestinal tract and the pancreas may also be regarded as possible organ sources of the hydrolase.

\section{Introduction}

Diadenosine tetraphosphate hydrolase is a nucleotide pyrophosphatase (EC 3.6.1.9) which occurs both in human plasma and serum. There are no differences of activity between the twio body fluids. The enzyme has been highly purified in our laboratory (1). It is a glycoprotein complex with a relative molecular mass of about $M_{\mathrm{r}}=200000$. For full enżyme activity the hydrolase needs divalent cations. The substrate specificity is not confined to diadenosine tetraphosphate $\left(\mathrm{Ap}_{4} \mathrm{~A}\right)$. The homologue dinucleotide diadenosine triphosphate $\left(\mathrm{Ap}_{3} \mathrm{~A}\right)$ is also cleaved yielding $\mathrm{ADP}$ and AMP as products. Several other nucleotides are attacked by the enzyme, but the preferred substrates of the pyrophosphatase are the dinucleotides $\mathrm{Ap}_{3} \mathrm{~A}$ and $\mathrm{Ap}_{4} \mathrm{~A}$ (1).
These dinucleotides have been found in human platelets in abundant amounts $(2,3)$. After activation of the platelets both dinucleotides are released into the surrounding medium $(2,3)$. Recently a physiological role of $\mathrm{Ap}_{3} \mathrm{~A}$ and $\mathrm{Ap}_{4} \mathrm{~A}$ in the haemostatic process has been claimed. $\mathrm{Ap}_{3} \mathrm{~A}$ has been shown to be an inducer of platelet aggregation, whereas $\mathrm{Ap}_{4} \mathrm{~A}$ inhibits this process (4). It is interesting that $A_{3} A$ itself does not trigger aggregation. The final trigger is ADP, which is generated from $\mathrm{Ap}_{3} \mathrm{~A}$ by the action of the nucleotide pyrophosphatase. Variations of its activity may be of significance for processes such as thrombosis and haemostasis.

Data on the catalytic activity concentration of the enzyme under normal as well as under various pathological conditions were hitherto lacking. The possible 
use of the enzyme as a tool in clinical biochemistry needs to be evaluated. In this pilot study we have focussed on inpatients of a medical clinic.

Since we measured enzyme activity in the present study with a bioluminescence assay, using $\mathrm{Ap}_{4} \mathrm{~A}$ as a substrate, we refer to the enzyme as diadenosine tetraphosphate hydrolase. The enzyme, however, does attack other nucleotides.

\section{Materials and Methods}

\section{Sera}

Fourty normal sera were obtained from healthy laboratory workers or from students. Their age ranged between 20 and 35 years (24 men, 16 women). Pathological sera were obtained from the University Hospital Erlangen-Nürnberg (medical clinic). Samples $(n=207)$ were collected within one month on four different days. The ratio between males and females was 1.5. The patients' age ranged from 19 to 88 years.

Further information on the patients was available in 182 of the 207 cases. In addition to the diagnosis, we utilized several laboratory parameters, such as serum protein, serum albumin, total bilirubin, amylase (serum), aspartate aminotransferase, alanine aminotransferase, $\gamma$-glutamyl transferase and lactate dehydrogenase. These values were taken from the medical records.

All the sera were stored frozen at $-20^{\circ} \mathrm{C}$. Diadenosine tetraphosphate hydrolase activities of the sera were determined within four weeks after receipt of the samples. There was no evidence of a loss of enzyme activity during this time.

Determination of diadenosine tetraphosphate hydrolase activity in human serum

We have used a slightly modified bioluminescence method which has already been described in detail (1). In principle, $\mathrm{Ap}_{4} \mathrm{~A}$ is cleaved by the hydrolase, yielding AMP and ATP as products. ATP is detected by the luciferin-luciferase reaction.

The reaction assay contained in a total volume of $0.5 \mathrm{ml}$ : $\mathrm{Ap}_{4} \mathrm{~A}$ as a substrate at $1 \mu \mathrm{mol} / \mathrm{l}, 25 \mathrm{mmol} / \mathrm{l}$ Hepes (pH 7.75), $3.8 \mathrm{mmol} / 1 \mathrm{MgCl}_{2}$ and $50 \mu \mathrm{l}$ of luciferin/luciferase (AMR; from LKB).

The reaction was started by the addition of 20 or $50 \mu$ of serum, which had been diluted $1: 10$ with Tris- $\mathrm{HCl} 50 \mathrm{mmol} / \mathrm{l}$ pH 8.0.

The increase of luminescence was monitored with a photomultiplier (Lumacounter Model 2080; Lumac Systems AG, Switzerland) every 30 seconds (integration mode). The increase of bioluminescence during the first minute after addition of enzyme (serum) was taken as initial velocity.

Since the luminescence of the AMR (per pmol of ATP) decreased with prolonged storage at room temperature, a calibration of the assay was necessary. This was performed by adding a standard quantity of ATP ( 2 pmol) to the reaction mixture described above, but in the absence of $\mathrm{Ap}_{4} \mathrm{~A}$ and serum. Activity determinations of four sera and one calibration experiment were run in parallel. The value of one calibration was taken as reference value. Ratios of the reference value and the values obtained from the other calibration experiments were used to . correct the serum enzyme activities.

\section{Statistics}

Mean values and standard deviations were computed. Gaussian distribution of the data was tested graphically. The values were plotted as a cumulative frequency histogram on probability paper. To assess the significance of differences between means the Student's t test or the test of Wilcoxon, Mann \&.Whitney (in the case of unequal variances) was used. Correlations between different laboratory parameters were ranalysed by calculation of the coefficients of correlation.

\section{Results}

Diadenosine tetraphosphate hydrolase assay

The linear relationship between diadenosine tetraphosphate hydrolase activity and the amount of serum in the assay is demonstrated in figure 1 . Two sera with different levels of enzyme activity were used. With both samples the hydrolase activities were linear up to $9 \mu \mathrm{l}$ of serum. For routine measurements enzyme activity wás determined with 2 and $5 \mu l$ of serum, respectively, both sample volumes therefore being within the linear range of the test. The linear relationship between the amount of enzyme present in the assay and the induced bioluminescence indicated that quench phenomena played no major role.

The imprecision of the test was evaluated as follows. About 80 sêra were pooled and stored in small portions at $-20^{\circ} \mathrm{C}$. Each determination was performed with a freshly thawed sample. Diadenosine tetraphos-

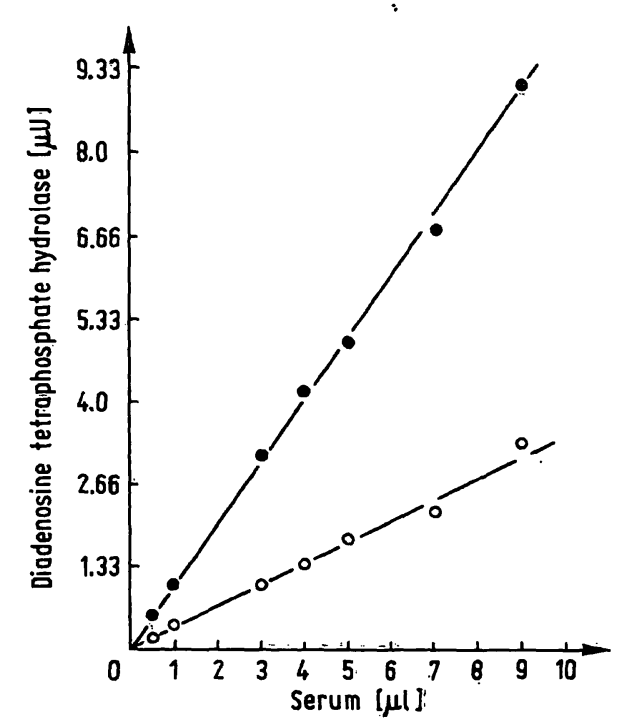

Fig. 1. Dependence of the measured catalytic activity concentration of diadenosine tetraphosphate hydrolase on the volume of serum used in the assay:

Two different sera were used in this experiment: one from a healthy donor with a normal enzyme catalytic concentration (open circles) and one serum obtained from an inpatient with an increased enzyme catalytic concentration (closed circles): 
phate hydrolase activities were measured on seven days within a period of five months. Five independent determinations were run per day. The imprecision (expressed as variation coefficients) on the seven days were $7.9 \%, 7.5 \%, 7.4 \%, 6.8 \%, 7.5 \%, 11.6 \%$ and $9.6 \%$, respectively. The corresponding mean values of the hydrolase activities were 117.4, 103.5, 110.9, $105.3,83.8,114.0$ and $96.2 \mathrm{mU} / 1$ of serum, respectively. The reproducibility from day to day $(11 \%)$ was a little worse than the reproducibility on one day (see above).

There was no evidence of a loss of enzyme activity during a storage period of 5 months (at $-20^{\circ} \mathrm{C}$ ).

A comparison of enzyme activities in serum and plasma revealed no differences. Also no differences were found in slightly haemolytic and non-haemolytic sera.

Diadenosine tetraphosphate hydrolase activities in normal and pathological sera

The activities of 40 healthy volunteers and of 207 inpatients from the local medical clinic were measured. The results are demonstrated as a relative frequency histogram in figure 2 . The mean values of the activities of the normal sera and of the pathological sera were $90.5 \pm 21.0( \pm$ SD) and $159.1 \pm 45.2$ $( \pm \mathrm{SD}) \mathrm{mU} / \mathrm{l}$ serum, respectively. Plotting of the data on probability paper revealed a Gaussian distribution for both groups. The differences between the mean activities were highly significant $(\mathrm{p}<0.001$, test of Wilcoxon, Mann \& Whitney).

Since both groups were heterogenous with respect to their age we wanted to rule out a possible influence of age on the enzyme activity (the age of the normal

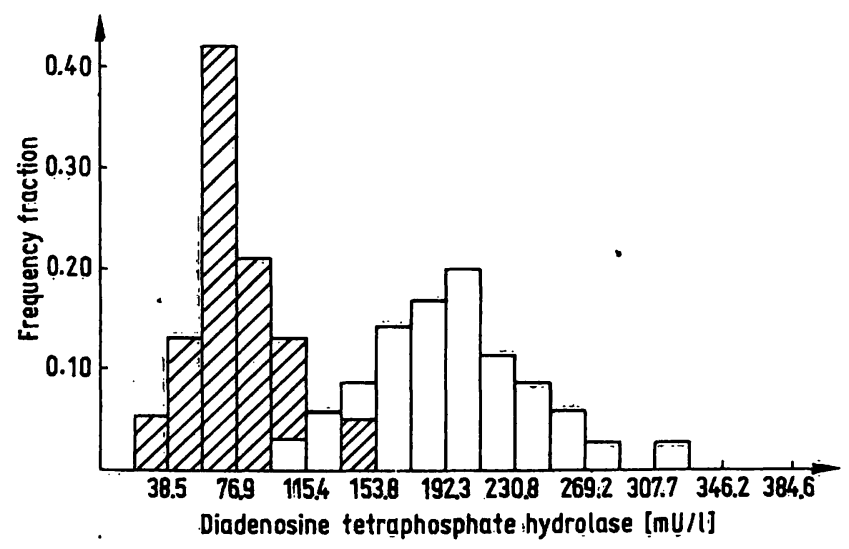

Fig. 2. Frequency histogram of diadenosine tetraphosphate hydrolase catalytic activity concentrations in sera of healthy donors (hatched bars, $n=40$ ) and in sera of inpatients (open bars, $\mathrm{n}=207$ ) donors ranged between 20 and 35 years, the age of the patients between 19 and 88). Therefore the reference group was compared only with those patients under the age of 35 . The results did not change essentially. On the contrary, the difference between both groups increased slightly (mean \pm SD of the patients: $180.1 \pm 37.7 \mathrm{mU} / 1$ of serum, $n=38$ ) (fig. 3). We then divided the group of inpatients into three classes of different age. The subgroups were designated as $A$ ( $\leqslant 45$ years), $B(46-60$ years) and $\mathrm{C}$ (>60 years). The mean enzyme activities in A, B and $C$ were $179.6 \pm 39.1( \pm \mathrm{SD}, \mathrm{n}=58) 154.6 \pm$ $47.1( \pm \mathrm{SD}, \mathrm{n}=68)$ and $145.5 \pm 43.8 \mathrm{mU} / \mathrm{l}$ of serum $( \pm S D, n=79)$, respectively. Thus a slight decrease of the hydrolase activity was observed with increasing age.

Furthermore there were no differences between males and females. The mean enzyme activities of the inpatients were $160.9 \pm 48.2( \pm \mathrm{SD}, \mathrm{n}=124)$ and $156.1 \pm 38.6 \mathrm{mU} / 1$ of serum $( \pm \mathrm{SD}, \mathrm{n}=83$ ), respectively. The healthy donors also showed no differences between the sexes.

When the diadenosine tetraphosphate hydrolase activities were compared with the diagnoses (fig. 4) increased as well as normal values were found with all disorders. Sera showing increased activities predominated in all groups. Patients with pancreatitis always had elevated levels of the hydrolase activity.

Diadenosine tetraphosphate hydrolase and other laboratory parameters

We compared the hydrolase activities in the serum with several other enzymes and with bilirubin (tab. 1). It is remarkable that $63.3 \%$ of the inpatients had

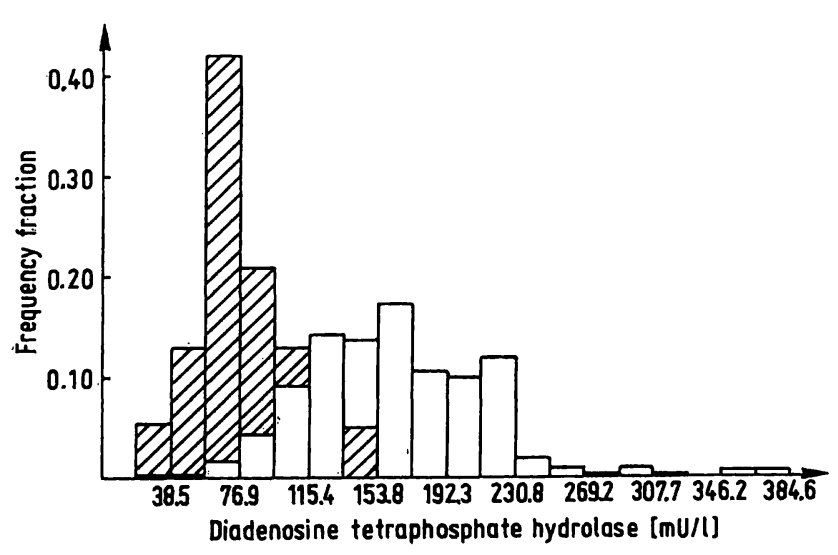

Fig. 3. Frequency histogram of diadenosine tetraphosphate hydrolase activities in sera of donors under the age of 35. Inpatients (open bars, $n=38$ ), healthy volunteers (hatched bars, $n=40$ ). 
Tab. 1. Comparison between diadenosine tetraphosphate hydrolase and other laboratory parameters.

Diadenosine tetraphosphate hydrolase activities were taken as increased, when the value was greater than $\bar{x}+2$ SD with regard to our reference group. The other parameters were evaluated according to the reference values used in the hospital laboratory.

$\mathbf{r}=$ coefficient of correlation, $\mathrm{n} . \mathbf{s} .=$ not significant, $\mathrm{n} . \mathrm{d} .=$ not detectable

\begin{tabular}{|c|c|c|c|c|c|}
\hline \multirow[t]{2}{*}{ Parameter } & \multirow{2}{*}{$\begin{array}{l}\text { Total } \\
\mathbf{n}\end{array}$} & \multicolumn{2}{|c|}{$\begin{array}{l}\text { Number of cases with an in- } \\
\text { creased level of the parameter }\end{array}$} & \multicolumn{2}{|c|}{$\begin{array}{l}\text { Correlations with diadenosine } \\
\text { tetraphosphate hydrolase }\end{array}$} \\
\hline & & $\mathbf{n}$ & $\%$ & $\mathbf{r}$ & (Significance) \\
\hline Diadenosine tetraphosphate hydrolase & 207 & 132 & 63.3 & - & - \\
\hline Aspartate aminotransferase & 180 & 61 & 33.9 & 0.10 & $(p<0.10)$ n.s. \\
\hline Alanine aminotransferase & 182 & 69 & 37.9 & 0.14 & $(\mathrm{p}<0.05)$ \\
\hline$\gamma$-Glutamyltransferase & 178 & 117 & 65.7 & 0.27 & $(p<0.001)$ \\
\hline Alkaline phosphatase & 179 & 70 & 39.1 & 0.20 & $(\dot{p}<0.005)$ \\
\hline$\alpha$-Amylase & & n.d. & n.d. & 0.04 & $(\tilde{\mathrm{p}}<0.40)$ n. s. \\
\hline Lactate dehydrogenase & 162 & 65 & 40.1 & 0.10 & $(\mathrm{p}<0.20)$ n. s. \\
\hline Bilirubin total & 175 & 46 & 26.3 & 0.05 & $(\mathrm{p}<0.30)$ n.s. \\
\hline
\end{tabular}

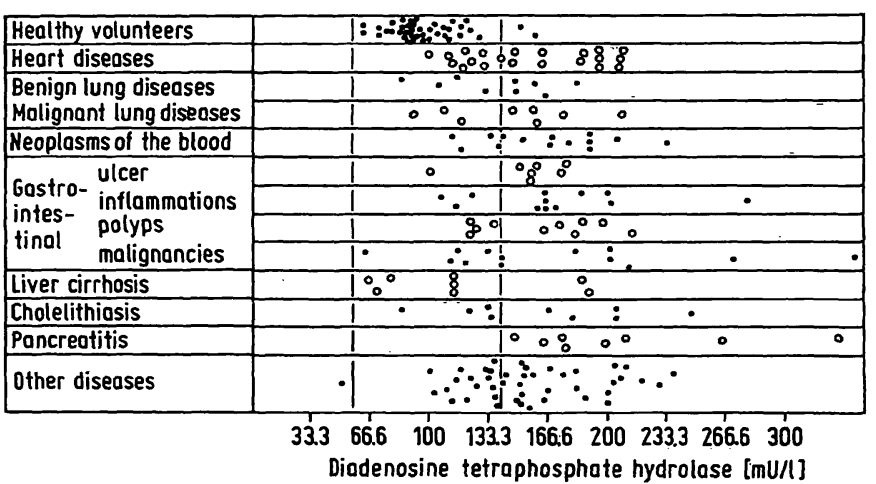

Fig. 4. Serum diadenosine tetraphosphate hydrolase catalytic activity concentrations in sera of healthy volunteers and 163 inpatients.

Results from 163 of 207 sera measured for enzyme catalytic activity concentrations could be related to a clear diagnosis. The group "heart diseases" mainly consisted of myocardial infarctions, coronary heart diseases and cardiac insufficiencies. Benign disorders of the lungs were pneumonia, emphysema and bronchitis. Acute and chronic myeloid leukaemia, Hodgkin's disease and malignant lymphomas were subsumed under "neoplasms of the blood". The group "other dieseases" contained 44 cases with 26 different disorders, both of benign and malignant nature. The dashed lines indicate the mean $\pm 2 \mathrm{SD}$ range of the catalytic concentrations from the normal donors. For reasons of clarity open and closed circles were used by turns.

increased levels of the diadenosine tetraphosphate hydrolase whereas the other values were elevated only in $26-40 \%$ of the cases, with the exception of $\gamma-$ glutamyl transferase, which also showed increased activities in about two thirds of the patients.

Comparison of the correlations between diadenosine tetraphosphate hydrolase and the seven other parameters (tab. 1), revealed small, but statistically significant correlations between the hydrolase and alkaline phosphatase, alanine aminotransferase, and $\gamma$-glutamyl transferase. The highest correlation was found with $\gamma$-glutamyl transferase. Of 117 patients with increased $\gamma$-glutamyl transferase $63.2 \%$ showed increased catalytic concentrations of the hydrolase.

It is noteworthy, however, that $67.2 \%$ of the 61 cases with normal $\gamma$-glutamyl transferase values also exhibited elevated activities of the diadenosine tetraphosphate hydrolase. Thus many patients with normal $\gamma$-glutamyl transferase, i. e. no sign of liver disease or accompanying liver reaction, had increased catalytic concentrations of the hydrolase. Analysis of the diagnoses of this group revealed that $53 \%$ suffered from gastrointestinal disorders (benign and malignant states of the stomach, small intestine and the colon). In the group of patients with increased levels of both enzyme activities, diseases of the gastrointestinal tract were only present in $12 \%$ of the cases. This was also true for the group with the constellation "increased $\gamma$-glutamyl transferase and normal diadenosine tetraphosphate hydrolase".

\section{Discussion}

Diadenosine tetraphosphate hydrolase is an enzyme which occurs in human serum and plasma in equally high amounts. It has been highly purified and partially characterized in our laboratory (1). The purpose of this study was to obtain information on the hydrolase levels in normals: Furthermore about 200 sera from inpatients of a medical clinic were measured in order to study the enzyme levels in various pathological conditions. Whether the diadenosine tetraphosphate hydrolase might be of value as a diagnostic tool in clinical biochemistry was a further question. 
This study clearly showed that inpatients had significantly increased levels of diadenosine tetraphosphate hydrolase activity as compared to the reference group. When relating enzyme activities with the diagnoses of the patients, increased hydrolase levels were found in quite different disorders. Thus elevation of the enzyme was not a specific marker for any particular disorder. At present, this enzyme has no obvious relevance as a diagnostic aid.

However, it should be stressed that about $64 \%$ of the patients showed increased values, whereas only $5 \%$ of the healthy donors had increased activity levels. Increased values of the diadenosine tetraphosphate hydrolase are not indicative of a specific disease, but elevated levels might be a general sign of illness. One should keep in mind that we have investigated a selected collective, namely typical patients of a medical clinic.

Nothing is known about the organ source of the elevated diadenosine tetraphosphate hydrolase levels. A direct approach to the investigation of organ distribution by measuring the hydrolase activities in homogenates of various organs would not give unambiguous results, because of the complexity of the $A_{4} A$ degrading activities; i. e. several cytosolic and particulate activities seem to coexist in a wide variety of tissues $(9,10)$. However, in this regard the present work may give some hints.

Since various diseases exhibited increased levels of the hydrolase one may assume that this enzyme is present in many organs and tissues. However, this assumption is not essential to the interpretation. For example, $\gamma$-glutamyl transferase was also increased in about two thirds of the cases, and it is known that this enzyme is almost only increased in diseases and reactions of the liver (and biliary tract) (5). This is explained by the fact that many diseases are accompanied by reactions of the liver.

This explanation could also hold true for the diadenosine tetraphosphate hydrolase. The elevations of enzyme activity would then be attributable to the liver. Several of our results support the hypothesis that the liver is a source of the hydrolase. When comparing the diadenosine tetraphosphate hydrolase with other laboratory parameters the close correlation with $\gamma$ glutamyl transferase was remarkable. $63.5 \%$ of the sera showing increased catalytic concentration of $\boldsymbol{\gamma}$ glutamyl transferase also had elevated activities of the $\mathrm{Ap}_{4} \mathrm{~A}$ hydrolase.

Besides the liver, other organs may act as a source of the serum enzyme activity. Of those patients showing a normal $\gamma$-glutamyl transferase activity, i.e. with no indication for liver cell damage (transaminases were also in the reference range), about $67 \%$ had increased levels of the diadenosine tetraphosphate hydrolase. This points to another organ or tissue as a source for increased hydrolase activities. It is remarkable that the analysis of the diagnoses revealed that over $50 \%$ of these patients suffered from gastrointestinal disorders.

Thus the gastrointestinal tract is very likely to be a further source of increased diadenosine tetraphosphate hydrolase activities in various states of disease. Another candidate is the pancreas. All cases of pancreatitis showed elevated hydrolase activity.

We have recently shown that human platelets contain $A p_{3} A$ and $A p_{4} A$ degrading activities (3). However, since the activities measured in serum and in plasma were identical, it appeared that platelets did not contribute to the hydrolase activity found in serum.

Human erythrocytes contain a phosphodiesterase activity which is strongly inhibited by $\mathrm{Zn}^{2+}$ (11). On the basis of this property, the erythrocyte enzyme is not identical with the diadenosine tetraphosphate hydrolase from serum, since the serum enzyme is strongly stimulated by $\mathrm{Zn}^{2+}$ (1). Furthermore there were no differences in enzyme activities between slightly haemolytic and non-haemolytic sera from the same donor.

A possible source of the serum diadenosine tetraphosphate hydrolase, however, might be the endothelial lining of blood vessels. An ectoenzyme which cleaves $\mathrm{Ap}_{3} \mathrm{~A}$ (which is also a substrate of the serum hydrolase) has recently been demonstrated on endothelial cells of pig aorta (12). We have confirmed and extended these results by detecting $A_{3} A$ and $A p_{4} A$ degrading activities on calf aortic endothelium (unpublished results). The glycoprotein structure of the serum hydrolase (1) suggests that this enzyme might originate from the membrane of vascular endothelial cells.

The variety of organs discussed here as a source of increased enzyme activities in the serum stimulates the question of the existence of isoenzymes. In analogy with enzymes such as alkaline phosphatase or lactate dehydrogenase, it is an obvious assumption that diadenosine tetraphosphate hydrolase also occurs in multiple molecular forms.

During the purification there were no indications for the existence of isoenzymes (1), but there are several possible reasons for this. Firstly, we isolated the enzyme from ACD-(acidum citricum/dextrose)-plasma. Since it is known that nucleotide pyrophosphatases are dependent on divalent cations for full activity 
some of the possible isoenzymes may have been inactivated by the complexing agent citrate. Secondly, it is possible that the separation techniques used were not sufficient to give a good resolution of isoenzymes. Thirdly, the enzyme was purified from plasma of healthy donors. Isozymes which are only formed under certain pathological conditions would have escaped our notice.

It should be mentioned that the highly purified enzyme (5000-fold) possessed a 5'-nucleotide phosphodiesterase activity (1). These enzymes are known to exist in different molecular forms in human serum $(6,7)$. For example, four activities were found in sera of healthy persons and under certain pathological conditions a fifth isozyme appeared. In a very recent paper even more activities were detected in the serum (8). We assume that at least one of the 5 -nucleotide phosphodiesterases is identical with the diadenosine tetraphosphate hydrolase. Further research is necessary to find out whether all the phosphodiesterases can cleave $A p_{4} A$ and whether there are diadenosine tetraphosphate hydrolases without any phosphodiesterase activity.

\section{Acknowledgement}

The authors gratefully acknowledge the continuous support by Prof. Dr. $W$. Kersten and the excellent technical assistance of Herbert Brönner. The work was supported by the Deutsche Forschungsgemeinschaft.

\section{References}

1. Lüthje, J, \& Ogilvie, A. (1985) Eur. J. Biochem. 149, 119127.

2. Flodgaard, H. \& Klenow, H. (1982) Biochem. J. 208, 737742.

3. Lüthje, J. \& Ogilvie, A. (1983) Biochem. Biophys. Res. Commun. 115, 253-260.

4. Lüthje, J. \& Ogilvie, A. (1984) Biochem. Biophys. Res. Commun. 118, 704-709.

5. Thomas, L. (1984) Labor and Diagnose, 2nd ed., pp 6974, Medizinische Verlagsgesellschaft Marburg/Lahn.

6. Tsou, K. C., Ledis, S., Lo, K. W. \& Mc Coy, M. G. (1973) J. Histochem. Cytochem. 21, 402.
7. Tsou, K. C. \& Lo, K. W. (1982) In: Methods in Cancer Research, Vol. XIX, 273-300, Academic Press, New York.

8. Lin, K. M., Ye, S. Y. \& Liu, Q. F. (1986) Int. J. Cancer $37,849-851$.

9. Cameselle, J. C., Costas, M. J., Sillerö, M. A. G. \& Sïillero, A. (1984) J. Biol. Chem. 259, 2879 - 2885.

10. Camesellè, J. C., Costas, M. J., Sillero, M. A. G. \& Sillero, A. (1982) Biochem. J. 201, 405-410.

11. Haugen, H. F. \& Skrede, S. (1977) Clin. Chem. 23, $1531-$ 1537.

12. Goldman, S. J., Gordon, E. L. \& Slakey, L. L. (1987) Circulation Res. 59, 362-366.

Dr. Jürgen Lüthje

Institut für Physiologische Chemie

Universität Erlangen-Nürnberg

Fahrstraße 17

D-8520 Erlangen 